\title{
Body Morphometrics in NAFLD Population
}

\author{
Harkeerat Dhami, Niharika Samala² \\ ${ }^{1}$ Indiana University School of Medicine, ${ }^{2}$ Indiana University School of Medicine, \\ Department of Medicine, Division of Gastroenterology and Hepatology.
}

\section{Introduction:}

NAFLD is one of the common causes of liver disease in the US and is commonly associated with metabolic syndrome. Among obese, prevalence of NAFLD is 70$90 \%$. We wanted to determine body morphometrics in NAFLD.

\section{Methods:}

All individuals presenting to Indiana University Hospital with NAFLD were approached to participate in cross-sectional study. All participants were offered beverage, diet (REAP) questionnaires and body composition analysis using InBody 570, which utilizes bioelectrical impedance.

\section{Results:}

Of the 321 NAFLD individuals enrolled, 256 completed body morphometric analysis. Mean age of the cohort was $51.58 \pm 13.54,58 \%$ were female, 297 White and had a mean BMI of 35.92 . $76 \%$ were obese, $48 \%$ had type 2 diabetes, $49.2 \%$ had hypertension, $38.6 \%$ had dyslipidemia, and $20.5 \%$ had obstructive sleep apnea. Despite having similar BMI, females had lower lean body mass (51.01 vs 70.51) and skeletal muscle mass (28.05 vs 39.70$)$, higher body fat mass (46.71 vs 41.04 ) and percent body fat (46.59 vs. 35.7). Regular coffee consumers had lower BMI (35.3 vs $38, p=0.038)$, but lower body fat mass (39.9 vs $46.2, p=0.01$ ), percent body fat (41.1 vs $44.4, p=0.05)$ and higher lean body mass $\%$ (58.8 vs $55.5, p=0.049)$. Processed meat consumption was associated with higher BMI (39 vs 35.3, $\mathrm{P}=0.01$ ), percent body fat ( 45.5 vs $42, \mathrm{p}=0.04$ ), and lower lean body mass percentage $(54.5$ vs $58.2, \mathrm{P}=0.04)$. Similar trends were seen with consumption of high sodium processed foods and watching television for $\geq 2$ hours/day.

\section{Conclusion:}

Among individuals with NAFLD, we saw a higher female preponderance, who were found to have unfavorable body morphometrics despite similar BMI as males. Consumption of high sodium processed food and meat and excess screen time have unfavorable, while regular coffee drinkers have favorable body morphometrics, which offer modifiable measures for risk factors associated with NAFLD. 